\title{
SU(2) Poincaré sphere: A generalized representation for multidimensional structured light
}

\author{
Yijie Shen $\odot,{ }^{1,2,3,}{ }^{*}$ Zhaoyang Wang, ${ }^{1,2}$ Xing Fu $\odot,{ }^{1,2}$ Darryl Naidoo $\odot,{ }^{4,5}$ and Andrew Forbes $\oplus^{5}$ \\ ${ }^{1}$ Key Laboratory of Photonic Control Technology (Tsinghua University), Ministry of Education, Beijing 100084, China \\ ${ }^{2}$ State Key Laboratory of Precision Measurement Technology and Instruments, Department of Precision Instrument, \\ Tsinghua University, Beijing 100084, China \\ ${ }^{3}$ Optoelectronics Research Centre, University of Southampton, Southampton SO17 1BJ, United Kingdom \\ ${ }^{4}$ CSIR National Laser Centre, P.O. Box 395, Pretoria 0001, South Africa \\ ${ }^{5}$ School of Physics, University of the Witwatersrand, Private Bag 3, Wits 2050, South Africa
}

(Received 18 January 2020; revised 14 August 2020; accepted 17 August 2020; published 9 September 2020)

\begin{abstract}
Structured light, as a general term for arbitrary states of amplitude, phase, and polarization in optical fields, is highly topical because of a myriad of applications it has fostered. A geometric description to graphically group classes of structured light has obvious benefits, with some notable advances in analogous Poincaré sphere (PS) mapping for both spin and orbital angular momentum (OAM), as well as ray-optical PS approaches for propagation-invariant fields, but all limited in dimensionality they can describe. Here we propose a generalized SU(2) PS for arbitrary dimensional structured light. The states on it represent extended families of beams with multidimensional ray-wave structures, accurately described by SU(2) symmetry groups. We outline how to construct this mapping theoretically, revealing insights into mode transformations involving OAM and geometric phase, and fully verify its efficacy experimentally. The generality of our approach is evident by the reduction to prior PS representations as special cases. We also demonstrate an extension of our approach to explain a more general high-dimensional vector beam. This construction naturally accounts for the salient topology of the classical PSs while bringing to more new degrees of freedom and dimensions for tailoring a larger variety of quantum-to-classical structured beams for a variety of applications.
\end{abstract}

DOI: 10.1103/PhysRevA.102.031501

The Poincaré sphere (PS) is a prominent graphical representation of light's polarization, where all the polarization states are mapped to its surface through the Stokes parameters as Cartesian coordinates [1]. This geometric connection provides not only remarkable physical insight but also great simplification of complex polarization problems, thus becoming a ubiquitous tool to treat structured light phenomena in numerous and varying fields. For example, in quantum optics we refer to it for revealing the evolution of two-dimensional (2D) entangled states [2]. In recent years, the PS has been generalized into various styles by introducing new degrees of freedom (DoFs) besides polarization so as to represent more general quantum states, which largely motivated its applications in quantum information and quantum-classical connection [3]. For instance, Padgett and Courtial replaced the polarization with orbital angular momentum (OAM) and created the OAM PS to interpret the evolution of transverse mode, i.e., the transformation between the first-order HermiteGaussian (HG) and Laguerre-Gaussian (LG) modes [4]. Then, such OAM PS was generalized for high-order HG and LG modes by the SU(2) basis eigenstate of coherent field $[5,6]$. Furthermore, if the eigenstates at the two poles are replaced into right- and left-handed circularly polarized OAM beams with the same topological charge but of opposite helicity, the high-order PS is obtained to reveal the spin-orbital coupled

\footnotetext{
*shenyj15@tsinghua.org.cn
}

vector vortex beams $[7,8]$. By replacing the states at the poles with two arbitrary OAMs in the high-order PS, the generated hybrid-order PS can represent an expanded set of vector vortex beams with general spin-to-orbital conversion $[9,10]$.

Beyond these PS models, a ray-optics PS was proposed to represent geometric rays by the points on its surface, and a curve rather than a point on which means a family of optical rays constituting a spatial mode, which can largely extend the set of represented structured Gaussian beams using a PS [11]. Apart from the polarization and structured beams, the PS was also modified to represent structured birefringence, where spatially varying birefringence is mapped on a hypersphere projected by the classical PS [12]. These adaptations of the PS model paved the way for extending newly hatched applications in imaging [13], communications [14,15], quantum information [3,16], and metamaterial designs [17-19].

In this Rapid Communication, we propose a generalized PS for representing the family of structured modes in a form of SU(2) coherent state [a well-defined superposed state by a set of SU(2) basis eigenstates [20]], encompassing the evolution of OAM and SU(2)-symmetric ray trajectories. We show that the prior PS representations are just specific cases of our higher-dimensional approach. In this new PS, the orthogonal bases (OBs) at the two poles are the right- and left-handed OAM states and the four mutually unbiased bases (MUBs) on the equator are the geometric modes with coherent state phases of $0, \pi / 2, \pi$, and $3 \pi / 4$. This new PS graphically simplifies the representation of complex structured light, playing 

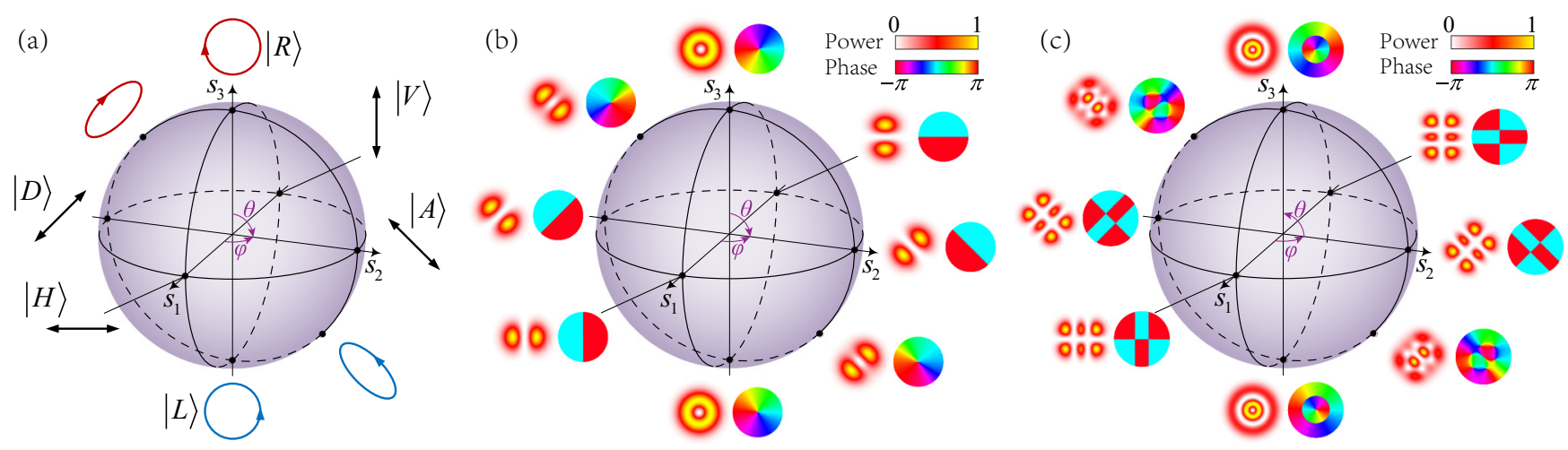

FIG. 1. (a) Classical PS for polarization states of plane wave. The poles represent right- and left-handed circular polarization, the equator linear polarization, and intermediate points between the poles and equator elliptical polarization. The northern and southern hemispheres separate right- (red) and left- (blue) handed ellipticity. States on antipodal points are orthogonal, and any polarization state is given as their linear combination. (b) OAM PS for OAM states of vortex modes. The poles represent right- and left-handed-OAM LG modes, the equator HG modes, and intermediate points between the poles and equator elliptical vortex mode. The northern and southern hemispheres separate rightand left-handed vortices. States on antipodal points are orthogonal, and any mode state is given as their linear combination. (c) HLG PS for topological transformation of HLG mode. The poles represent the high-order LG modes of opposite OAM, the equator high-order HG modes, and intermediate points between the poles and the equator HLG mode. The northern and southern hemispheres separate positive and negative total topological charge. States on antipodal points are orthogonal, and any mode state is given as an SU(2) transformation of eigenmodes. In (b) and (c), the insets show the transverse pattern (left) and phase (right) of the represented modes of selected points on the PSs.

as a salient toolkit to enrich the technologies of optical manipulation and quantum information, particularly pertaining to multidimensional states of classically entangled light.

To begin, consider an arbitrary polarization (spin) state

$$
\left|\psi_{\mathrm{p}}\right\rangle=\cos (\theta / 2) e^{-i \varphi / 2}|R\rangle+\sin (\theta / 2) e^{i \varphi / 2}|L\rangle,
$$

which can be mapped on a sphere with Cartesian coordinates $\left(s_{1}, s_{2}, s_{3}\right)=\left(s_{0} \cos \varphi \sin \theta, s_{0} \sin \varphi \sin \theta, s_{0} \cos \theta\right)$, where the right- and left-handed circular polarizations, $|R\rangle$ and $|L\rangle$, respectively, are at the two poles as the OBs, and the horizontal, antidiagonal, vertical, and diagonal linear polarizations, $|H\rangle$, $|A\rangle,|V\rangle$, and $|D\rangle$, respectively, are uniformly distributed at the equator as the four MUBs, as Fig. 1(a) shows. An analogous description is given in terms of OAM mode states [4]:

$$
\left|\psi_{0}\right\rangle=\cos (\theta / 2) e^{-i \varphi / 2}\left|\ell_{1}\right\rangle+\sin (\theta / 2) e^{i \varphi / 2}\left|\ell_{2}\right\rangle .
$$

As the formation of basic OAM PS $\left(\ell_{1}=-\ell_{2}=1\right)$ [4], the two OBs are the right- and left-handed LG modes, and the four MUBs are horizontal, antidiagonal, vertical, and diagonal HG modes, as shown in Fig. 1(b). The tensor product of polarization PS and OAM PS, $\left|\psi_{\mathrm{p}}\right\rangle \otimes\left|\psi_{\mathrm{o}}\right\rangle$, can generate the high-order PS (for $\ell_{1}=-\ell_{2}$ ) [7] and hybrid-order PS [9] representing various vector vortex beams. In these classical PS models, the two states on any pair of antipodal points are orthogonal to each other and can play as two eigenstates to linearly express any state on the same PS with superposition coefficient related to the angular coordinates.

In order to represent the higher-order modes, the oscillator model is usually used to express the beam mode as the eigenstate of coherent field [21], which is given by

$$
\left|\psi_{n, m, l}\right\rangle=\frac{\left(b_{1}^{\dagger}\right)^{n}}{\sqrt{n !}} \frac{\left(b_{2}^{\dagger}\right)^{m}}{\sqrt{m !}} \frac{\left(b_{3}^{\dagger}\right)^{l}}{\sqrt{l !}}|0,0,0\rangle,
$$

where the creation operators yield an $\mathrm{SU}(2)$ transformation:

$$
\left[\begin{array}{l}
b_{1}^{\dagger} \\
b_{2}^{\dagger} \\
b_{3}^{\dagger}
\end{array}\right]=\left[\begin{array}{ccc}
e^{-i \theta / 2} \cos (\varphi / 2) & e^{i \theta / 2} \sin (\varphi / 2) & 0 \\
-e^{-i \theta / 2} \sin (\varphi / 2) & i \theta / 2 \cos (\varphi / 2) & 0 \\
0 & 0 & 1
\end{array}\right]\left[\begin{array}{c}
a_{1}^{\dagger} \\
a_{2}^{\dagger} \\
a_{3}^{\dagger}
\end{array}\right],
$$

where $a_{i}^{\dagger}(i=1,2,3)$ are the creation operators of a transversely symmetric harmonic oscillator at $x, y$, and $z$ axes, $(n, m)$ are the transverse mode indices at the $(x, y)$ plane, $l$ is the longitudinal mode index, and $|0,0,0\rangle$ now represents the fundamental mode Gaussian beam. This generalized form can be mapped on a new PS by the same angular coordinate just with a reversed $\theta$, as shown in Fig. 1 (c) for $(n, m)=(2,1)$, which is actually the Hermite-Laguerre-Gaussian (HLG) mode PS recently proposed as the higher-order extension of the fundamental OAM PS [6]. In fact, all prior PS representations are contained in this formulation: when $(n, m)=(0,1)$ or $(1,0)$, the HLG PS [Fig. 1(c)] reverts back to the wellknown OAM PS [Fig. 1(b)]. States on a pair of antipodal points on the HLG PS are the two orthogonal eigenstates and any state on the PS is the result of an SU(2) transformation with corresponding angular coordinates as parameters. In the HLG PS, the angle $\varphi$ does not introduce a new structure in the mode pattern, it simply rotates the pattern. In order to generalize the formulation further, we thus want to replace the function of the parameter $\varphi$ in order to introduce more controllable structured beams with PS representation.

Since the HLG mode mapped on a PS can be expressed as an SU(2) basis eigenstate, we use the SU(2) coherent state mode as a superposition of a set of eigenmodes [22]. To further generalize the PS model, we set the HLG modes as eigenstates and rewrite the wave packet of the SU(2) coherent state as

$$
\left|\psi_{n, m, l}^{N, P, Q}\right\rangle=\frac{1}{2^{N / 2}} \sum_{K=0}^{N} e^{i K \phi}\left(\begin{array}{l}
N \\
K
\end{array}\right)^{1 / 2}\left|\psi_{n+Q K, m, l-P K}\right\rangle,
$$



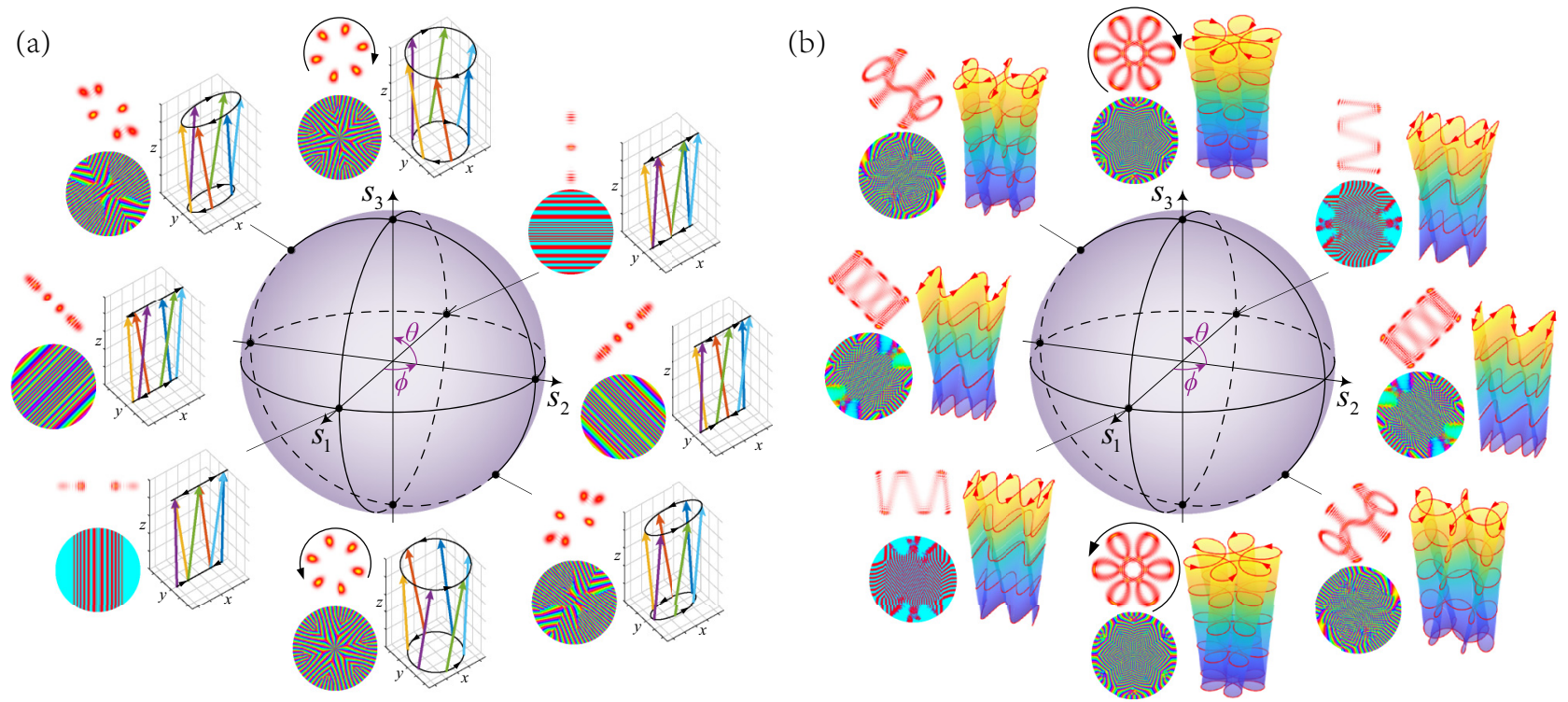

FIG. 2. (a) SU(2) PS for multipath geometric modes. The poles represent the vortex geometric modes with opposite OAM, the equator planar geometric modes, and intermediate points between the poles and the equator the elliptical geometric modes. (b) SU(2) PS for Lissajoustrochoidal parametric surface modes. The poles represent the vortex trochoidal modes with opposite OAM, the equator Lissajous modes, and intermediate points between the poles and the equator the topological modes between the Lissajous and trochoidal parametric surface. For both (a) and (b), the northern and southern hemispheres separate positive and negative total OAM. States on antipodal points are orthogonal, and any mode state is given as an SU(2) superposition of HLG eigenmodes. The insets show the transverse pattern (upper left), phase (lower left), and classical trajectory (right) of the represented modes of selected points on the PSs. The arrows in the classical trajectories mark the directions of photon behavior.

where $\phi$ is the coherent state phase and $P / Q$ ( $P$ and $Q$ are coprime integers) is the ratio of transverse and longitudinal mode spacings resulting into frequency degeneracy, i.e., the transverse ( $x$ direction) and longitudinal modes of various eigenmodes should fulfill the condition of coherent superposition. As a characteristic of the coherent state, the behavior of the wave packet should be coupled with the classical movement trajectory of the corresponding Hamiltonian, the classical trajectory of which can be expressed by [21]

$$
\left[\begin{array}{l}
x_{b} \\
y_{b} \\
z_{b}
\end{array}\right]=\left[\begin{array}{ccc}
e^{-i \theta / 2} \cos (\varphi / 2) & e^{i \theta / 2} \sin (\varphi / 2) & 0 \\
-e^{-i \theta / 2} \sin (\varphi / 2) & e^{i \theta / 2} \cos (\varphi / 2) & 0 \\
0 & 0 & 1
\end{array}\right]\left[\begin{array}{c}
x_{a} \\
y_{a} \\
z_{a}
\end{array}\right],
$$

where $\left(x_{b}, y_{b}, z_{b}\right)$ represents the trajectory of classical movement yielded by the Hamiltonian $\hat{H}=\sum_{i=1}^{3}\left(b_{i}^{\dagger} b_{i}+1 / 2\right) \hbar \omega_{i}$ $\left(\omega_{i}\right.$ are the oscillator component frequencies, $i=1,2,3=$ $x, y, z)$. Specifically when $m=0,\left(x_{b}, y_{b}, z_{b}\right)$ represents the multiray trajectory located on a ruled uniparted hyperboloid and the $\mathrm{SU}(2)$ coherent state mode is a multipath geometric mode located on the rays; when $|m|>0$, the fundamental beams on the multipath rays would be replaced by high-order mode beams, resulting into multiaxis HLG beams [23]. This enables us to map the $\mathrm{SU}(2)$ coherent state onto a new generalized PS with angular coordinates $(\phi, \theta)$ covering the original $(\varphi, \theta)$, namely, a SU(2) PS, as shown in Fig. 2(a) for the geometric mode for the case of $P / Q=1 / 6$ $(n=12, m=0, N=8)$. This representation reveals physical significance, for example, the topological evolution of the geometric mode coupled with the classical ray trajectory and OAM can be vividly revealed, where the evolution from the equator to the poles corresponds to the process of introducing OAM to the mode. Moreover, when $Q \rightarrow \infty$, the geometric mode can be approximated to an HLG eigenstate [24] and the SU(2) PS can be reduced into an HLG PS.

More generally, if the oscillator components at the transverse plane are coupled into frequency degeneracy [21], the $\mathrm{SU}(2)$ coherent state evolves into

$$
\left|\psi_{n, m, l}^{N, P, p, q}\right\rangle=\frac{1}{2^{N / 2}} \sum_{K=0}^{N} e^{i K \phi}\left(\begin{array}{l}
N \\
K
\end{array}\right)^{1 / 2}\left|\psi_{n+p K, m+q K, l-P K}\right\rangle
$$

where $(p, q)$ is a pair of integers yielding $Q=p+q$ and $\omega_{2} / \omega_{1}=p / q$, which can represent more generalized structured beams that are similarly mapped on the SU(2) PS with the angular coordinate $(\phi, \theta)$. In this case, the classical trajectory $\left(x_{a}, y_{a}, z_{a}\right)$ represents a Lissajous parametric surface as shown in Fig. 2(b) for $P / Q=1 / 4$ and $p / q=-1 / 5(n=$ $20, m=40, N=6$ ). In this generalized SU(2) PS, a point on the equator represents a coherent state mode located on a three-dimensional (3D) Lissajous parametric surface with a transverse wave packet located on a specific two-dimensional (2D) Lissajous curve $[21,25]$ With the point moving to the poles, i.e., the introduction of OAM, the mode can be topologically transformed into the mode located on a 3D trochoidal parametric surface with transverse wave packet located on a specific 2D trochoid [21,26]. In the SU(2) PS, the coherent states on a pair of antipodal points are orthogonal to each other, and any coherent state is the $\mathrm{SU}(2)$ wave-packet superposition with the corresponding HLG modes as eigenstates on the same point of the PS. Thus the OAM PS and HLG PS are 


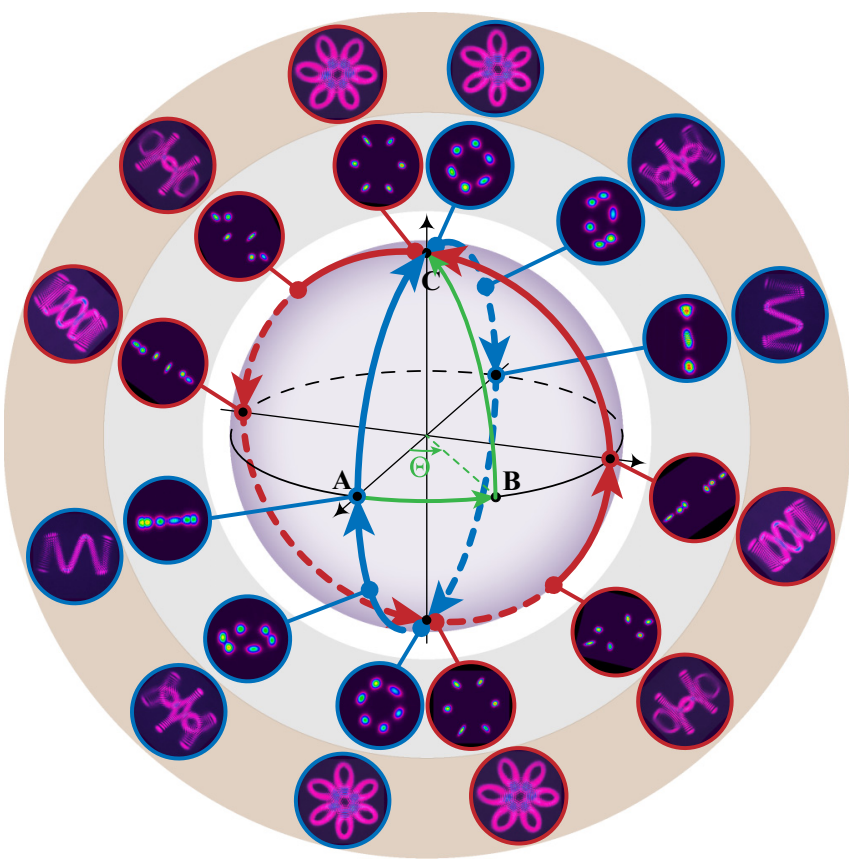

FIG. 3. Experimental generation of modes on specific routes (marked in red and blue) on the SU(2) PS, including multipath geometric modes (in gray region) and Lissajous-trochoidal modes (in brown region). The green route starts from point $\mathrm{A}(0,0)$ to $\mathrm{B}(\Theta, 0)$, then to the north pole $\mathrm{C}(\Theta, \pi / 2)$.

the special cases of the general SU(2) PS (see Supplemental Material Secs. I and II [27]).

It is pertinent to discuss some properties of the SU(2) PS akin to the classical PS. In the polarization PS or OAM PS, the points approaching a pole along different paths on the surface result in the same circular mode but with different phases, yielded by the effect of geometric phase [28]. For instance, as for the PS shown in Fig. 3, we consider two modes represented by the same north pole $\mathrm{C}$; the first mode $\psi_{\mathrm{C}}$ is directly transported from point $\mathrm{A}$ along the zero meridian, and the second $\psi_{\widetilde{\mathrm{C}}}$ firstly travel from $\mathrm{A}$ to $\mathrm{B}$ along the equator with a longitude angle of $\Theta$ and then to $\mathrm{C}$ along the corresponding meridian. Modes $\psi_{\mathrm{C}}$ and $\psi_{\widetilde{\mathrm{C}}}$ represent the same circular mode but with a phase difference of $\Theta / 2$. This implies that the circular mode of $\psi_{\widetilde{\mathrm{C}}}$ is obtained via rotating the mode $\psi_{\mathrm{C}}$ by an angle $\Theta / 2$. The two indistinguishable circular modes at pole $\mathrm{C}$ arise from the rotation-invariant amplitude pattern (the circular polarization or the annular LG mode). But this rotational invariance is just a special case $(Q \rightarrow \infty)$ of $\mathrm{SU}(2)$ symmetry, where the mode is invariant after rotating through an angle of $2 \pi / Q$. Thus for the $\mathrm{SU}(2) \mathrm{PS}$, besides the original geometric phase $\Theta / 2$, another rotation angle of $\Theta / Q$ should be added (for the degeneracy ratio of $P / Q$ ). Thus the geometric phase for our SU(2) PS is consistent with the traditional theory (see Supplemental Material Sec. III [27]).

The angles $\phi$ and $\theta$ also have clear physical significance. The coherent state phase $\phi$ is related to the topology of the periodic trajectory of the oscillating geometric modes in a degenerate laser cavity, and can be controlled by selective pumping [22,29]. The angle $\theta$ is related to the OAM controlled by an astigmatic mode converter [30-32].

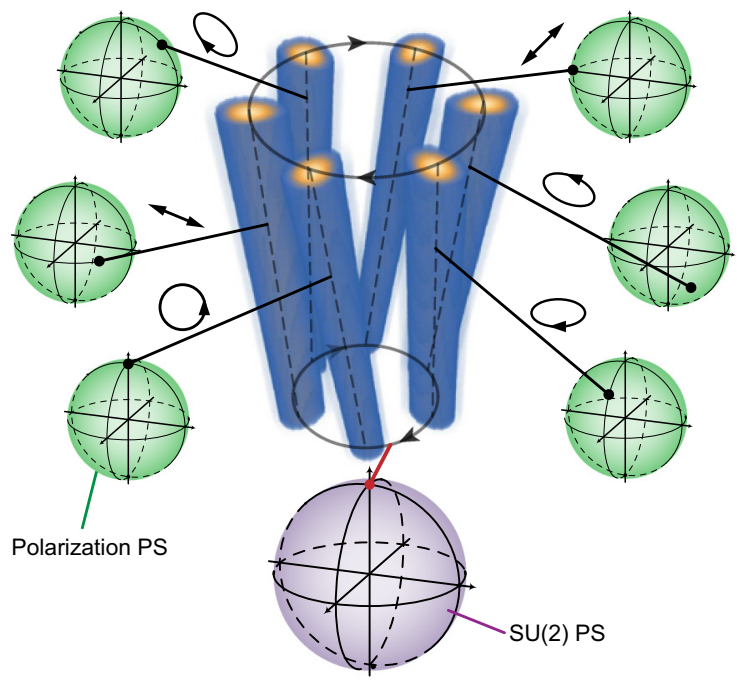

FIG. 4. Generalized SU(2) structured vector beam: the spatial wave-packet structure is determined by an SU(2) PS (purple) and the light on each ray state can be modulated into an arbitrary state of polarization on the polarization PS (green).

These insights lend themselves to immediate experimental implementation: we can controllably generate the modes around a circle across the poles on the SU(2) PS, as the blue ( $\phi=0$ or $2 \pi)$ and red routes $(\phi=\pi / 2$ or $3 \pi / 2)$ show in Fig. 3 , with the experimental results for both multipath geometric modes and Lissajous-trochoidal modes (see Supplemental Material Sec. IV [27]). Whereas, the selected pumping method is hard to realize a continuous change of $\phi$ [33]. Thus it is still a target in the future to fully and controllably generate all modes on the SU(2) PS.

Further, our framework can make a clear transition from the conventional cylindrical vector beams (represented by the prior high-order PS [7,9]) to more general multi-DoF and high-dimensional vector beams. For example, modulation of the polarization on each ray path of an SU(2) PS mode leads to novel vector beams not yet observed, as shown in Fig. 4. Such fields encompassing higher dimensions cannot be described within existing PS frameworks and breaks the conventional paradigm of the $2 \mathrm{D}$ vector beam so entrenched in our thinking.

This SU(2) PS geometric representation can open up a myriad of applications. For example, the 3D ray-wave structure makes clear new controllable DoFs in both the transverse and axial directions, rather than the classical transversely symmetrical and propagation-invariant OAM modes, which is a special case of our new model. In other words, this framework allows one to see more generalized OAM evolution in a 3D wave packet and provides a clear roadmap to multi-DoF high-dimensional states of structured light. To give an example from quantum structured light, conventional OAM-entangled photon pairs with LG modes comprise a oneDoF infinite-dimensional state $\sum a_{\ell}|\ell\rangle|-\ell\rangle$ [34] because the angular position is undetectable due to the rotation invariance of traditional OAM modes. Using the SU(2) PS framework, we find the potential to move off this position on our PS allowing the angular position DoF to be used, for OAM-entangled photons in two DoFs $\sum a_{\ell, \phi}|\ell, \phi\rangle|-\ell,-\phi\rangle$, 
for higher-capacity quantum and classical communications [14-16]. Similar approaches will surely inspire advances in the traditional applications of such structured light, e.g., realizing generalized optical tweezers with more complex particle control [35,36], and laser machining of more exotic shapes on functional materials [37], to name but a few. Another interesting extension would be to control the caustics to shape the coupled light beam [38], akin to Airy beams [39], which has a specific parabolic caustic ray structure, while our SU(2) PS mode can break this limit with more general 3D ray-coupled properties. The ray-wave duality mode also has direct relevance to many other fundamental physical effects such as the trajectory-spin coupling in the optical spin-Hall effect [40] and ray-wave chaos in microlasers [41,42].

In summary, our SU(2) PS is a very general representation for structured beams, reducing to the existing PSs under special conditions. The topological evolution of planar and vortex
SU(2) geometric modes can be fully mapped on the SU(2) PS. It represents the evolution of not only $2 \mathrm{D}$ mode patterns but also 3D wave packets coupled with classical trajectories for multidimensional structured light. Moreover, it also has the potential to be extended to include spin-orbital coupling. Therefore, the SU(2) PS proposed and demonstrated here offers a useful tool to graphically represent a large family of structured beams carrying OAM, with more DoFs and dimensions for further extending new theories and applications of structured light manipulation in quantum-to-classical optics.

This work is funded by National Key Research and Development Program of China (2017YFB1104500), National Natural Science Foundation of China (61975087), Natural Science Foundation of Beijing Municipality (4172030), Beijing Young Talents Support Project No. (2017000020124G044), and Marie S.-Curie MULTIPLY Fellowship (GA713694).
[1] H. Poincaré, Theorie Mathematique de la Lumiere (GauthiersVillars, Paris, 1892), Vol. 2.

[2] I. Bengtsson and K. Życzkowski, Geometry of Quantum States: An Introduction to Quantum Entanglement (Cambridge University Press, Cambridge, UK, 2017).

[3] E. Toninelli, B. Ndagano, A. Vallés, B. Sephton, I. Nape, A. Ambrosio, F. Capasso, M. J. Padgett, and A. Forbes, Adv. Opt. Photonics 11, 67 (2019).

[4] M. J. Padgett and J. Courtial, Opt. Lett. 24, 430 (1999).

[5] G. Agarwal, J. Opt. Soc. Am. A 16, 2914 (1999).

[6] M. R. Dennis and M. A. Alonso, Philos. Trans. R. Soc. London A 375, 20150441 (2017).

[7] G. Milione, H. I. Sztul, D. A. Nolan, and R. R. Alfano, Phys. Rev. Lett. 107, 053601 (2011).

[8] D. Naidoo, F. S. Roux, A. Dudley, I. Litvin, B. Piccirillo, L. Marrucci, and A. Forbes, Nat. Photonics 10, 327 (2016).

[9] X. Yi, Y. Liu, X. Ling, X. Zhou, Y. Ke, H. Luo, S. Wen, and D. Fan, Phys. Rev. A 91, 023801 (2015).

[10] R. C. Devlin, A. Ambrosio, N. A. Rubin, J. B. Mueller, and F. Capasso, Science 358, 896 (2017).

[11] M. A. Alonso and M. R. Dennis, Optica 4, 476 (2017).

[12] A. Vella and M. A. Alonso, Opt. Lett. 43, 379 (2018).

[13] N. A. Rubin, G. D'Aversa, P. Chevalier, Z. Shi, W. T. Chen, and F. Capasso, Science 365, eaax1839 (2019).

[14] A. Trichili, C. Rosales-Guzmán, A. Dudley, B. Ndagano, A. B. Salem, M. Zghal, and A. Forbes, Sci. Rep. 6, 27674 (2016).

[15] B. Ndagano, B. Perez-Garcia, F. S. Roux, M. McLaren, C. Rosales-Guzman, Y. Zhang, O. Mouane, R. I. HernandezAranda, T. Konrad, and A. Forbes, Nat. Phys. 13, 397 (2017).

[16] A. Forbes and I. Nape, AVS Quantum Sci. 1, 011701 (2020).

[17] Z. H. Jiang, L. Kang, T. Yue, H.-X. Xu, Y. Yang, Z. Jin, C. Yu, W. Hong, D. H. Werner, and C.-W. Qiu, Adv. Mater. 32, 1903983 (2020).

[18] E. Maguid, I. Yulevich, M. Yannai, V. Kleiner, M. L. Brongersma, and E. Hasman, Light: Sci. Appl. 6, e17027 (2017).

[19] J. P. Balthasar Mueller, N. A. Rubin, R. C. Devlin, B. Groever, and F. Capasso, Phys. Rev. Lett. 118, 113901 (2017).

[20] V. Bužek and T. Quang, J. Opt. Soc. Am. B 6, 2447 (1989).

[21] Y. Chen, J. Tung, P. Tuan, and K. Huang, Ann. Phys. 529, 1600253 (2017).
[22] Y. F. Chen, C. H. Jiang, Y. P. Lan, and K. F. Huang, Phys. Rev. A 69, 053807 (2004).

[23] P. Tuan, Y. Hsieh, Y. Lai, K. Huang, and Y. Chen, Opt. Express 26, 20481 (2018).

[24] Y. Chen, S. Li, Y. Hsieh, J. Tung, H. Liang, and K. Huang, Opt. Lett. 44, 2649 (2019).

[25] K. J. Górska, A. J. Makowski, and S. T. Dembínski, J. Phys. A: Math. Gen. 39, 13285 (2006).

[26] T. H. Lu, Y. C. Lin, Y. F. Chen, and K. F. Huang, Phys. Rev. Lett. 101, 233901 (2008).

[27] See Supplemental Material at http://link.aps.org/supplemental/ 10.1103/PhysRevA.102.031501 for more detailed demonstrations.

[28] E. Cohen, H. Larocque, F. Bouchard, F. Nejadsattari, Y. Gefen, and E. Karimi, Nat. Rev. Phys. 1, 437 (2019).

[29] J. C. Tung, P. H. Tuan, H. C. Liang, K. F. Huang, and Y. F. Chen, Phys. Rev. A 94, 023811 (2016).

[30] G. F. Calvo, Opt. Lett. 30, 1207 (2005).

[31] Y. Shen, X. Fu, and M. Gong, Opt. Express 26, 25545 (2018).

[32] Y. Shen, X. Yang, D. Naidoo, X. Fu, and A. Forbes, Optica 7, 820 (2020).

[33] Y. Shen, X. Yang, X. Fu, and M. Gong, Appl. Opt. 57, 9543 (2018).

[34] M. Erhard, R. Fickler, M. Krenn, and A. Zeilinger, Light: Sci. Appl. 7, 17146 (2018).

[35] M. Padgett and R. Bowman, Nat. Photonics 5, 343 (2011).

[36] M. Woerdemann, C. Alpmann, M. Esseling, and C. Denz, Laser Photonics Rev. 7, 839 (2013).

[37] J. Ni, C. Wang, C. Zhang, Y. Hu, L. Yang, Z. Lao, B. Xu, J. Li, D. Wu, and J. Chu, Light: Sci. Appl. 6, e17011 (2017).

[38] A. Zannotti, C. Denz, M. A. Alonso, and M. R. Dennis, Nat. Commun. 11, 3597 (2020).

[39] N. K. Efremidis, Z. Chen, M. Segev, and D. N. Christodoulides, Optica 6, 686 (2019).

[40] X. Ling, X. Zhou, K. Huang, Y. Liu, C.-W. Qiu, H. Luo, and S. Wen, Rep. Prog. Phys. 80, 066401 (2017).

[41] S. Bittner, S. Guazzotti, Y. Zeng, X. Hu, H. Y1lmaz, K. Kim, S. S. Oh, Q. J. Wang, O. Hess, and H. Cao, Science 361, 1225 (2018).

[42] H. Cao, R. Chriki, S. Bittner, A. A. Friesem, and N. Davidson, Nat. Rev. Phys. 1, 156 (2019). 